LA GROUNDED THEORY

\section{Y LA INVESTIGACIÓN}

CUALITATIVA EN

COMUNICACIÓN Y

MARKETING

\section{Pere Soler Pujals}

\author{
Profesor Titular \\ Universitat Autònoma de Barcelona. Urgel, 280 Sobreático, 2a \\ 08036, Barcelona (España) - Tlfn.: (+34) 934391449 - Email: \\ pere.soler@uab.es
}

\section{Betsaida Fernández Gómez}

Profesora Asociada

Universitat Autònoma de Barcelona. Rius i taulet 12, Parets del Vallès, 08150, Barcelona (España) - Tlfn.: (+34) 647908033 Email: betsaida.fernandez@uab.cat

\section{Resumen}

La Grounded Theory, desarrollada por Barney G. Glaser y Anselm L. Strauss, ha tenido en los últimos años una importante repercusión en el campo de la investigación cualitativa, aplicada principalmente en clínica y educación. Consideramos que su metodología es perfectamente aplicable en investigación comercial, con un rigor científico difícilmente asumible con las metodologías cualitativas que habitualmente se emplean.

\section{Palabras clave}

Teoría Fundamentada, Teoría Anclada, estudios de mercado, investigación

comercial, investigación cualitativa, comunicación. marketing, metodología de análisis cualitativo, consumidor

Key Words

Grounded Theory, consumer research, business research, qualitative research, communication, marketing, qualitative analysis methodology, consumer

\section{Abstract}

Grounded Theory, developed by Barney G. Glaser and Anselm L.

Strauss, has had in the last years an important impact on the qualitative

research field, mainly applied to clinical and educational research. We consider that its methodology is perfectly applicable to business research, with a scientific rigor hardly achievable with the qualitative methodologies that are usually used. 


\section{Introducción. El problema de la investigación cualitativa}

El problema de las investigaciones cualitativas es el problema de la investigación científica en ciencias sociales. E. Nagel (1968) afirma que no existen leyes contrastadas en las ciencias sociales, ya que es imposible la realización de experimentos controlados.

En todo experimento controlado es necesario poder manipular las variables que entran en el mismo cuantas veces sea necesario. En investigación social esto es muy difícil, por no decir imposible. La investigación cualitativa según E. Ortega (2007) tomando como referencia la obra de Hussey y Hussey (1997) nos dice que es subjetiva, humanista e interpretativa. Para otros autores como Cook y Reichard (1982) es un método de observación sin control, sus datos estadísticamente no son representativos del universo y, por tanto, son estudios aislados y no generalizables. Se genera una gran cantidad de información y el estudio proviene, en la gran mayoría de los casos, de unos pocos sujetos y de diferentes fuentes (Alvarez-Gayou, 2005).

Sobre la validez y fiabilidad en un análisis de contenido cualitativo, E. López Aranguren (1986) opina que un análisis de contenido es válido en la medida en que las descripciones e inferencias realizadas se mantienen en pie cuando se las confronta con datos obtenidos de forma independiente. La problemática actual en la investigación en marketing es que raramente se pueden encontrar, según diferente material recopilado, dos técnicos que empleen una misma metodología o proceso de análisis. Veamos algunos de los principales problemas:

- Generalmente en estos trabajos no existe una definición de conceptos que estudiar.

- La descripción de la metodología de análisis no es precisa. A otro investigador le es difícil repetirla y comprobar empíricamente sus resultados.

- Los investigadores cualitativos no acaban de ponerse de acuerdo para solventar las diferentes definiciones de conceptos que existen en estos estudios, ni para establecer un lenguaje común.

Consideramos que las hipótesis formuladas deberían ser verificadas en otras observaciones o datos. Apenas existen comunicaciones en este sentido.

Muchas de las investigaciones cualitativas con los métodos que habitualmente se utilizan en el campo del marketing difícilmente pasarían los filtros básicos o los requisitos necesarios para una comunicación de resultados, ateniéndose a las normas del manual de la APA o a las esta- 
blecidas por la American Psychological Association. En apoyo de la metodología cualitativa cabe decir, sin embargo, que las investigaciones exploratorias y sus análisis son importantes variables explicativas que pueden ser investigadas sistemáticamente. El problema se presenta al querer probar las hipótesis formuladas, quedando como único recurso el probarlas en nuevas formulaciones $\mathrm{y}$ verificaciones, siempre $\mathrm{y}$ cuando, y este es el principal peligro, no se ofrezcan diferentes explicaciones.

Como nos dicen Festinger y Katz (1987) "una codificacion fiable exige que se use el plan en la misma forma, la misma definición operacional de categorías, el mismo marco de referencia, el mismo grado de diferenciación, el mismo nivel de atención hacia los detalles... a través de todo el proceso de análisis”.

Revisando toda esta serie de problemas, creemos que la investigación en marketing debe buscar otras metodologías de análisis de datos como, por ejemplo, la Teoría Fundamentada o Grounded Theory formulada por los sociólogos Barney G. Glaser y Anselm L. Strauss de la Universidad de Columbia, la cual ha sido aplicada con muy buenos resultados en el campo clínico y de la educación (Charmaz, 1999; Altrichter, 1989; Chenail y Maione, 1999; Kinach, 1996; Shavarini, 1997; Miles y Huberman, 1994). Desde hace unos años, también se está empezando a aplicar en el ámbito de los negocios (David Douglas, Business School, Staffordshire University, UK,
2004; Walter D. Fernández, The Australian National University, Australia, 2004; Bruce H. Rowlands, Griffith University, Australia, 2005;...), y para el estudio de los hábitos de consumo (Pettigrew, S. 2002; Decrop, A. y Snelders, D., $2003 ; \ldots)$.

La Teoría Anclada o Grounded Theory nace en los años 60 a partir de una investigación desarrollada por los sociólogos Barney Glaser y Anselm Strauss, que en aquellos momentos estaban colaborando en un estudio acerca de pacientes moribundos. Dicha colaboración les llevó a escribir el libro "Conciencia de morir" (Awareness of Dying).

A partir de aquí desarrollan el método comparativo constante que más tarde se conocería como Teoría Anclada o muestreo teórico. Este muestreo teórico fue desarrollado como una metodología sistemática. Actualmente la Teoría Anclada ha tenido una gran resurgimiento y la vemos aplicada en varios campos y áreas de investigación (Bryant, 2002).

La Teoría Anclada, como nos dice el mismo A. Strauss (1989), "es una metodología de investigación de naturaleza exploratoria con énfasis en la generación y desarrollo de teorías que especifican los fenómenos y las condiciones para manifestarse". Glaser (1992) la describe como "una metodología de análisis, unida a la recogida de datos, que utiliza un conjunto de métodos, sistemáticamente aplicados, para generar una teoría inductiva sobre un área substantiva”. 
Sobre esta definición cabe destacar, por tanto, que estamos ante una metodología que busca la generación de teoría emergente, de carácter inductivo; no se trata de una metodología orientada a la descripción, sino a la conceptualización. Para Goulding (2002) la Teoría Anclada es un método cualitativo, teniendo muchas similitudes con otros métodos cualitativos como la etnografía (estudio descriptivo e interpretativo de la realidad del grupo). Las fuentes de información son las mismas: entrevistas, observación de campo y uso de documentos de todo tipo (diarios, cartas, autobiografías, periódicos y otros medios audiovisuales).

Según Strauss, esta metodología puede utilizarse para analizar cualquier tipo de dato, aunque hace especial referencia a entrevistas y observaciones de campo, y por ello describe la Grounded Theory como un "estilo de hacer análisis cualitativo" (Strauss, 1987).

Al igual que en otras estrategias de investigación cualitativa, en la Teoría Anclada el desarrollo del proceso investigativo no es lineal. La dinámica del trabajo es tanto metódica como recursiva, porque los investigadores han de categorizar sistemáticamente los datos y limitar la teorización hasta que los patrones de los datos emerjan de la operación de categorización. Este método requiere la recolección de información, la categorización abierta, la elaboración de memos analíticos que interpreten los datos obtenidos, la identificación de una(s) categoría(s) de núcleo (codificación selectiva), el ordenamiento de los métodos analíticos y la escritura de la teoría.

La mayor diferencia entre la Teoría Anclada y otras perspectivas cualitativas de investigación es su énfasis en el desarrollo de la teoría. Los investigadores pueden lograr varios niveles de teoría cuando utilizan sus procedimientos, independientemente de si ésta ha sido construida dentro del estilo de permanente interrelación entre la recolección de información y el análisis teórico y con el imperativo de la verificación de las hipótesis que van emergiendo. Podemos destacar 5 diferencias principales entre la Grounded Theory y la Entrevista en Profundidad o la Reunión de Grupo:

\section{Cuadro $^{\circ} 1$. Diferencias entre la Grounded Theory y la Entrevista en Profundidad o la Reunión de Grupo}

\begin{tabular}{|l|l|}
\hline $\begin{array}{l}\text { Reunión de Grupo - } \\
\text { Entrevista en Profundidad }\end{array}$ & Grounded Theory \\
\hline $\begin{array}{l}\text { Aceptada en el campo de la } \\
\text { investigación en comunica- } \\
\text { ción y marketing }\end{array}$ & $\begin{array}{l}\text { No aceptada universale- } \\
\text { mente }\end{array}$ \\
\hline Muestra fija & $\begin{array}{l}\text { Muestra por satura- } \\
\text { ción. Cada caso riguro- } \\
\text { samente seleccionado }\end{array}$ \\
\hline $\begin{array}{l}\text { Resultado inmediato en el } \\
\text { tiempo }\end{array}$ & $\begin{array}{l}\text { Se desconoce tiempo } \\
\text { requerido (finalización } \\
\text { investigación) }\end{array}$ \\
\hline $\begin{array}{l}\text { De las preguntas se obtienen } \\
\text { respuestas }\end{array}$ & $\begin{array}{l}\text { Las respuestas pueden } \\
\text { llevarnos a nuevas } \\
\text { preguntas clave }\end{array}$ \\
\hline De la teoría a los datos & $\begin{array}{l}\text { La teoría emerge de los } \\
\text { datos }\end{array}$ \\
\hline $\begin{array}{l}\text { No existe teoría para investi- } \\
\text { gar de nuevo }\end{array}$ & $\begin{array}{l}\text { Buscar teoría que gene- } \\
\text { ralice un área conceptual } \\
\text { entera }\end{array}$ \\
\hline
\end{tabular}

Fuente: Elaboración propia 


\section{Objetivos}

Muchos autores han escrito sobre la Teoría Fundamentada como forma de investigación cualitativa, y en la mayoría de ocasiones coinciden en el carácter diagnóstico de la misma. La Grounded Theory, permite "investigar, diagnosticar y poner tratamiento” contemporáneamente, debido a la cercanía entre los datos y la teoría.

Y no es casualidad que, tratándose de una teoría orientada al problema y cuya fuente principal de datos es la propia situación de conflicto, gran cantidad de autores la hayan utilizado para estudiar las ciencias sociales y todo aquello que concierne al ser humano y su comportamiento. De hecho, Strauss y Glaser remarcan de esta teoría su finalidad de solventar el problema entendiendo la acción a través de la perspectiva del agente. Es por esta razón, y por su gran capacidad metodológica, que la Grounded Theory puede ser de gran ayuda y extensa aplicación en el ámbito del marketing.
La Teoría Anclada es capaz de ofrecer, por tanto, una multiplicidad de alternativas para comprender realidades del mercado y del consumidor. Es un método que debe ser aplicado con dosis de imaginación y creatividad, sin perder nunca de vista su sistematicidad y rigurosidad, como se ha demostrado en otros campos, especialmente el clínico y el de la enseñanza.

Consideramos que aplicando su metodología podemos lograr importantes avances en la investigación del comportamiento del consumidor, ya que gracias a ella es posible descubrir el punto de vista del actor principal: un conocimiento más profundo de las razones de compra y consumo de determinados productos, los perfiles de los consumidores, las actitudes, motivaciones, hábitos y estilos de vida, atributos del producto, definición del posicionamiento, estrategias de comunicación, conocimiento de la competencia, etc.

\section{Metodología. La metodología de la GT aplicada a la investigación comercial}

La Teoría Anclada, siendo un método de investigación relativamente nuevo en el campo de la comunicación y el marketing, ofrece una gran oportunidad de investigación cualitativa (historia oral, etnometodología,...), pudiéndose asimismo combinar con enfoques cuantitativos. La aplicación de la Teoría Anclada en marketing cuenta con unos antecedentes muy diversos, a título de ejemplo podemos citar alguno de ellos: Ichikawa \& Santos (2001) presentan un programa para post-graduados en administración (Anpad). Martins (1997) analiza 126 tesis y disertaciones en el periodo de 
1980 a 1993 de las escuelas consideradas de referencia en el área de administración y marketing en América del Sur. Bacellar (2005) nos comenta la problemática de los profesores para la enseñanza del marketing con el método grounded. Partington (2000) propone el método grounded como estudio para entender el comportamiento gerencial proponiendo simplificación $y$ customización en su aplicación.

Muchos autores han escrito sobre la Teoría Fundamentada como forma de investigación cualitativa, y en la mayoría de ocasiones coinciden en el carácter diagnóstico de la misma. La Grounded Theory permite "investigar, diagnosticar y poner tratamiento” contemporáneamente, debido a la cercanía entre los datos y la teoría. Y no es casualidad que, tratándose de una teoría orientada al problema y cuya fuente principal de datos es la propia situación de conflicto, gran cantidad de autores la hayan utilizado para estudiar las ciencias sociales y todo aquello que concierne al ser humano y su comportamiento. De hecho, Strauss y Glaser remarcan de esta teoría su finalidad de solventar el problema entendiendo la acción a través de la perspectiva del agente. Es por esta razón, y por su gran capacidad metodológica, que la Grounded Theory puede ser de gran ayuda y extensa aplicación en el ámbito del marketing.

La Teoría Anclada es capaz de ofrecer, por tanto, una multiplicidad de alternativas para comprender realidades del mercado, de las organizaciones institucionales, em- presas y del consumidor. Es un método que debe ser aplicado con dosis de imaginación y creatividad, sin perder nunca de vista su sistematicidad y rigurosidad, como se ha demostrado en otros campos, especialmente el clínico y el de la enseñanza.

Consideramos que aplicando su metodología podemos lograr importantes avances, tanto en el conocimiento del consumidor como de las necesidades en comunicación de la empresa o de las instituciones, ya que gracias a ella es posible descubrir : un conocimiento más profundo de las razones de compra y consumo de determinados productos o servicios, los perfiles de los consumidores, las actitudes, motivaciones, hábitos y estilos de vida, atributos del producto, definición del posicionamiento $\mathrm{y}$ centrar el insight, estrategias de comunicación de todo tipo (financiera,corporativa, interna...). Investigar la credibilidad de la comunicación empresarial e institucional y la reputación de estas marcas, empresas o instituciones, conocimiento de la competencia (benchmark), etc. Sus posibilidades son enormes.

La Teoría Anclada es un método que tiene características muy específicas. Como puntos fuertes podemos citar los siguientes:

- Se fundamenta en datos empíricos, siendo estos datos muy próximos a la realidad (ya que la recolección de datos y el análisis deben ser simultáneos y la teoría puede surgir en cualquier etapa de la investigación - Charmaz, 2006) 
- Puede ser una herramienta muy eficaz para el estudio del comportamiento del consumidor, ya que está basada en el análisis constante de este comportamiento (el método comparativo constante es lo que confiere a esta metodología su continua validez).

- Salir al campo con un referencial teórico todavía en formación permite al investigador buscar nuevas perspectivas, teorías e hipótesis difíciles de formular en la investigación cualitativa tradicional, donde unas entrevistas en profundidad, reunión de grupo o focus group parecen ilusoriamente suficientes para tomar decisiones importantes para la empresa.

En un mercado donde cada día se demuestra más importante la venta de intangibles y el peso de la identidad de marca, la Teoría Anclada puede mostrar a los directores de comunicación y marketing como son realmente sus consumidores y con qué se sienten identificados.

Es evidente que existen algunas dificultades en su aplicación a la investigación en comunicación y marketing, como son las divergencias entre los autores y todos los debates posteriores acerca de la teoría. Es un proceso difícil con un tiempo indefinido de ejecución (y por tanto también un coste indefinido). Sin embargo, es de esperar que los resultados de esta metodología de investigación convenzan a los responsables de comunicación y marketing de sus múltiples ventajas.

Nuestra propuesta de las diferentes fases analíticas de construcción para la investigación en comunicación y marketing es la siguiente:

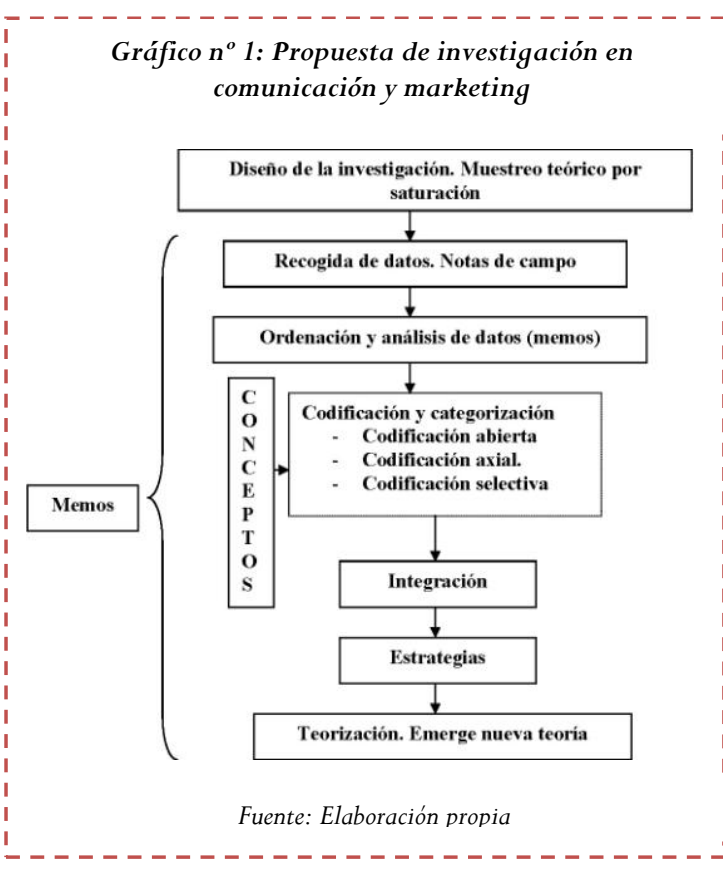

En la metodología de investigación, el trabajo debe iniciarse en su primera fase con una muestra teórica (Theoretical Sampling), es decir, individuos, situaciones y eventos idealizados para el proceso de análisis, que irán siendo indicados por la teoría que vaya emergiendo a lo largo de la investigación. Su representatividad debe verse más en términos de relevancia ("amplitud del fenómeno estudiado”) que en términos probabilísticos. Las situaciones y grupos deben elegirse en función de su 
pertenencia, aunque resulta imposible fijarlo de antemano.

Goulding (1999) explica que surgen a lo largo del estudio informadores que orientan los esfuerzos de recopilación de información hacia nuevos lugares. El grupo teórico como base para la creación de hipótesis y desarrollo de teorías debe emerger de los datos, por tanto la muestra debe ser flexible a las necesidades cambiantes de la investigación y el investigador debe ser capaz de analizar constantemente los datos obtenidos con el objetivo de encontrar aquellas situaciones/ individuos (muestra) que aporten nuevos datos a la teoría.

Nuevos grupos pueden ser lógicamente incorporados al proceso. Según Laperrière (1997), el muestreo teórico inicial es determinado por la pregunta de investigación ${ }^{i}$ y es continuamente remodelado para responder a nuevos interrogantes que surgen del análisis. Existe una saturación teórica cuando no emerge nueva información de los datos recogidos. Glaser y Strauss (1967) definen que no hay un único formato para describir la teoría de este proceso.

En la fase de recogida de datos o de familiarización, se trata de recopilar una base de datos (como una recopilación de notas de campo) y de leer los datos recogidos las veces necesarias para poderlos analizar y determinar su interés para ser utilizados. Es en estas notas donde se descubren y etiquetan variables y sus interrelaciones. Este proceso nos permitirá formular la idea base y las preguntas a responder (hay que recordar que las teorías se derivan de los datos). Es en esta fase donde debe empezarse a construir los "memos", que son simplemente ideas teóricas anotadas separadamente de las notas de campo que deben consultarse y revisarse en el transcurso de toda la investigación. Gracias a ellos podremos encontrar en sus registros categorías, conceptos y propiedades y sus interrelaciones entre categorías e incluso hipótesis de trabajo.

El proceso de nombrar o etiquetar las cosas, las categorías y las propiedades es conocido como fase de codificación y categorización.

La codificación realizada nunca será la definitiva, ya que al igual que hemos comentado en la muestra, pueden emerger algunas nuevas categorías y éstas también pueden hacer variar las anteriores. Sin embargo, sí es aconsejable mantener una existencia de claves con sus descripciones (crear un codebook), con todas las notas disponibles para ser revisadas en cualquier momento, que nos serán muy útiles para los informes. Debemos señalar que un código es lo más próximo a un concepto teórico, entendiendo el concepto teórico como la unidad básica de análisis, dado que la teoría se desarrolla a partir de la conceptualización de datos. Las categorías son superiores en nivel y más abstractas que los conceptos que representan. El agrupamiento de conceptos forma categorías. 
Cuando hablamos de codificación abierta nos referimos a la parte del análisis involucrado en identificar, nombrar, categorizar y describir los fenómenos encontrados en el texto. Respondería a preguntas como: ¿qué es esto? ¿qué se está mencionando aquí?. Es una etapa muy pesada para el investigador, ya que se trata de un proceso analítico donde se identifican las categorías más generales que emergen de los datos brutos. Esta codificación se basará tanto en los datos en bruto como en la experiencia, incluido el conocimiento de la literatura sobre la cuestión (Strauss, 1987). Son seleccionadas las palabras-clave que generarán los conceptos que serán la base de la próxima fase del proceso (Goulding, 2001). El investigador da aquí nitidez a la teoría.

La codificación axial es el proceso de relacionar códigos (categorías y propiedades) vía una combinación inductiva y deductiva, reabriendo la búsqueda para validarla o no. Los conceptos seleccionados son analizados y reorganizados, con el objetivo de extraer una idea central y sus subordinaciones. En esta fase se puede volver al campo, elegir más elementos de análisis o volver al grupo inicial para una nueva búsqueda de datos.

En la codificación selectiva o core categorization se reducen las categorías iniciales y se busca la variable de núcleo o categoría central. A partir de aquí se buscan las relaciones de las diferentes categorías con la denominada variable de núcleo, no descartándose nunca la recogida de nuevos datos. Esta categoría central establece el paradigma de la teoría (Goulding, 2001). El proceso se acaba según Corbin y Strauss (1990) cuando no se puede añadir ningún dato más al proceso de análisis y categorización.

Cada concepto tiene sus propiedades, aplicándolas de la misma forma que se hace en la Teoría Anclada. Las propiedades son cualidades, atributos, explican lo que está ocurriendo (Ej. En un estudio sobre geles para la limpieza corporal pueden surgir propiedades como: La piel pierde lípidos grasas integrantes de la piel-. Con lo que se reseca la piel y puede irritarse) ${ }^{\mathrm{ii}}$.

Posteriormente, llevaremos a cabo la integración, es decir, la puesta en relación de las categorías según las analogías e intereses que comporten. En este punto tendremos en cuenta la triangulación, si existe combinación de distintos métodos o fuentes de datos.

En cuanto a las estrategias de acción, son las actividades resueltas que los agentes llevan a cabo en respuesta al fenómeno estudiado. La estrategia desde un punto de vista operativo es la idea a seguir para conseguir los objetivos. En comunicación y marketing una estrategia es siempre una solución a un problema determinado. Las consecuencias son lógicamente los resultados de la aplicación del diseño. 


\section{Conclusiones}

Estudios sistematizados y apoyados en la metodología de la Grounded Theory consideramos que tienen mucho futuro en la investigación cualitativa comercial en comunicación y marketing. Basta una pequeña revisión de las herramientas que se han venido utilizando hasta ahora y cuya problemática hemos comentado al principio de este artículo para darnos cuenta de que la investigación cualitativa tiene en la Grounded Theory un campo de muy amplio reco- rrido, donde la calidad de las investigaciones se hace patente en su confiabilidad al demostrar que las operaciones de un estudio se pueden repetir con los mismos resultados. Probando así su validez externa de generalización y la validez interna de procedimiento mediante el establecimiento de relaciones causales por medio de las cuales ciertas condiciones se demuestra que conducen a otras.

\section{Referencias}

ÁLVAREZ-GAYOU, J. L. (2005). Cómo hacer investigación cualitativa. Fundamentos y metodología. Barcelona, Edit. Paidós.

CARRERO, V., SORIANO, R. M., y TRINIDAD, A. (2006). Teoría fundamentada $<$ Grounded theory $>$. La construcción de la teoría a través del análisis interpretacional. Madrid, Edit. CIS (Centro de Investigaciones Sociológicas).

CHARMAZ, K. (2006). Constructing grounded theory. A practical guide through qualitative analysis. London, Edit. Sage.

CORBIN, J. y STRAUSS, A. L. (1990). Basics of qualitative research: Grounded theory procedures and techniques. London, Edit. Sage Publications.

GLASER, B. G. (1978). Theoretical sensitivity: Advances in the methodology of grounded theory. Mill Valley, CA, Edit. The Sociology Press.

GLASER, B. G. (1992). Basics of grounded theory analysis: Emergence vs. forcing. Mill Valley, CA, Edit. The Sociology Press.

GLASER, B. G. y STRAUSS, A. L. (1967). The discovery of grounded theory strategies for qualitative research. Chicago, Edit. Aldine Pub.
GOULDING, C. (1999). Consumer research, interpretive paradigms and methodological ambiguities. "European Journal of Marketing", 33(9/10), pp. 859-873.

GOULDING, C. (2001). Grounded theory: A magical formula or a potential nightmare. "The Marketing Review", 2(1), pp. 21-34.

GOULDING, C. (2005). Grounded theory, ethnography and phenomenology. "European Journal of Marketing”, 39(3/4), pp. 294-308.

HUSSEY, J. \& HUSSEY, R. (1997). Business research: A practical guide for undergraduate and postgraduate students. London, Edit. Macmillan Press.

JONES D., MANZELLI H., y PECHENY M. (2004). Grounded theory. Una aplicación de la teoría fundamentada a la salud. "Cinta de Moebio: Revista Electrónica de Epistemología de Ciencias Sociales”, (19), pp. 56-67.

LOCKE, K. (2001). Grounded theory in management research. London, Edit. Sage Publications.

LOFLAND, L. (2001). Handbook of ethnography. London, Edit. Sage Publications. 
PARTINGTON, D. (2000). Building grounded theories of management action. "British Journal of Management”, 11, pp. 91-102.
STRAUSS, A. L. (1987). Qualitative analysis for social scientists. Cambridge, Edit. University Press.

\section{NOTAS}

Agradecemos la participación en la búsqueda de material y la presentación de trabajos sobre este tema del alumnado de Doctorado del Departamento de Comunicación Audiovisual y Publicidad de la Universidad Autónoma de Barcelona. Alumnado: Joana Blass, L.A. Davidovich, Cristina Villar, D. do Espítu Santo, Cinara M. Battisti, Eder Pérez, Pere Navalles, Eunyce Pena, Ricardo Navarro.

${ }^{\mathrm{i}}$ Antonio Trinidad, Virgina Carrero y Rosa M ${ }^{\mathrm{a}}$ Soriano, 2006

${ }^{\text {ii }}$ Existe una cierta dificultad en diferenciar lo que es una propiedad de lo que es una categoría (Jones D., Manzelli H., Pecheny M., 2004). 